\title{
Analysis of PAPR on DFT-OFDMA Systems
}

\author{
Surabhi Singh \\ Department of Electronics \& Communication \\ Jaypee University, A-10, Sector-62, Noida (U.P.)- \\ India
}

\author{
Shambhavi Mishra \\ Department of Electronics \& Communication \\ Jaypee University, A-10, Sector-62, Noida (U.P.)- \\ India
}

\begin{abstract}
OFDM is a broadband wireless technology which supports data rates in excess of $100 \mathrm{Mbps}$. In addition to this, OFDM has some limitations as it requires strict frequency synchronization and low peak to average power ratio. So considering DFT-OFDMA, which performance in terms of PAPR, is equivalent to SC-FDMA. This paper represents the suitability of using OFDMA in the up-link for high-data-rate scenarios in local area by considering target performance metrics PAPR. The proposed design shows PAPR performance varies depending on the subcarrier allocation method. It has been also shown that performance of the PAPR by DFT-spreading technique with IFDMA and LFDMA, varying with roll-off factor $\alpha$ of the RC (Raised-Cosine) filter. Effect of the number of subcarriers on PAPR performance is also discussed in this paper.
\end{abstract}

\section{Keywords}

Complementary cumulative distribution function(CCDF) ,Distributed FDMA(DFDMA), Interleaved(IFDMA), Localized FDMA(LFDMA)

\section{INTRODUCTION}

OFDM main principle is to divide the data stream to be transmitted into a series of number of narrowband orthogonal subcarriers. That is means of an inverse fast Fourier transform, which allows for an increased symbol period. In particular, for a minimum PAPR, low number of subcarriers are preferable.

OFDM requires an expensive and inefficient power amplifier in the transmitter because of the high Peak-to-Average Power Ratio (PAPR) of the multi-carrier signal. Each user is allocated a subset of subcarriers in OFDMA, hence each user must be scheduled by system. So group of subcarrier is defined as subchannel. Depending on how subcarrier are allocated to construct each subchannel, resource allocation strategies classified as-

Distributed: All of the RBs allocated to the every user are randomly distributed over the entire bandwidth. Localized: All of the RBs are allocated to the every user in a contiguous manner. By implementation, it has been found that localized allocation of RBs will be considered in order to give good results to keep the PAPR benefits of the SCFDM signal.

However, this scheme leads to a constraint on the flexibility of the resource allocation in a multi-user scenario. The paper is organized as follows. PAPR characterize by CCDF in OFDM signals is given in section II. Proposed performance of SCFDMA with different subcarrier location is given in section III, simulation and effect of changing and subcarrier on PAPR IV. Variation in PAPR accordingly $\alpha$ and subcarrier. Section $\mathrm{V}$ concludes the paper.

\section{COMPLEMENTARY CUMULATIVE DISTRIBUTION FUNCTION (CCDF)}

The CCDF is a parameter to characterize the peak power statistics of a digitally modulated OFDM signal. The CCDF of PAPR information about the percentage of OFDM signals that have PAPR above a particular level. To better approximate the PAPR of a continuous time OFDM signal, the discrete time OFDM signal is to be obtained by L times oversampling. The oversampled discrete time OFDM signal can be obtained by performing $\mathrm{L} \mathrm{N}$ point IFFT on the data block with (L-1)N zero padding as follows[4]:

$$
\mathrm{x}[\mathrm{n}]=\frac{1}{\sqrt{N}} \sum_{k=0}^{N-1} X_{\mathrm{k}} \exp \left(\frac{j 2 \pi \mathrm{mm}}{L N}\right) \quad 0 \leq n \leq N L-1
$$

The probability distribution of complex OFDM signals with large $\mathrm{N}$ is a complex Gaussian distribution given by following relation

$\mathrm{P}_{\mathrm{r}}\{\mathrm{x}[\mathrm{n}]\}=\frac{1}{\sqrt{2 \pi \sigma^{2}}} \exp ^{\left(\frac{-\mathrm{x}^{2}[\mathrm{n}]}{2 s^{2}}\right)}$

where $\operatorname{Pr}\{$.$\} denotes the probability distribution function.$

The real and imaginary parts of the complex OFDM signal has Gaussian distribution therefore the magnitude of OFDM signal has Rayleigh distribution. The cumulative distribution function (CDF) of PAPR of an OFDM signal with $\mathrm{N}$ subcarriers is given by

$$
\begin{aligned}
& \operatorname{CDF}\left(\gamma_{0}\right)=\operatorname{Pr}\left(\operatorname{PAPR}(x[\mathrm{n}]) \leq \gamma_{0}\right) \\
& =\left(1-\exp \left(-\gamma_{0}\right)\right)^{\mathrm{N}}
\end{aligned}
$$

Where $\gamma_{0}$ is the threshold value of PAPR.

Hence, complementary cumulative distribution function $(\mathrm{CCDF})$ of PAPR becomes CCDF $\left(\gamma_{0-}\right)=\operatorname{Pr}(\operatorname{PAPR}(\mathrm{x}[\mathrm{n}])>$ $\left.\gamma_{0}\right)$

$=\left(1-\exp \left(-\gamma_{0}\right)\right)^{\mathrm{N}}$

When oversampling is done by a factor $\mathrm{L}$, the CCDF of OFDM signal (3.20) changes to

$\mathrm{CCDF}=\left(1-\exp \left(-\gamma_{0}\right)\right)^{\mathrm{LN}}$

Fig. 1 shows the plots for the CCDF of the OFDM signals for various oversampling factor (L). Here, an OFDM system with QPSK modulation and $\mathrm{N}=256$ subcarrier is assumed in the simulation. It can be easily observed from the Fig. 1, that the PAPR of the discrete-time OFDM signal increases as increase the oversampling factor $(\mathrm{L})$, but for $\mathrm{L} \geq 4$, the increase in the CCDF of PAPR is very less, hence, an oversampling 
factor $\mathrm{L}=4$ seems to be adequate for a good estimate of PAPR for a continuous time OFDM signal.

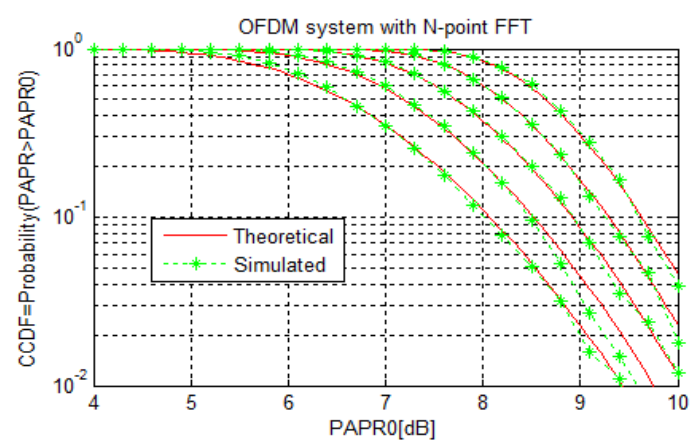

Fig.1 CCDFs of OFDM signals with $\mathrm{N}=64,128,256,512$, and 1024.

Subcarriers are mutually orthogonal hence overlapping between them is allowed which provide highly spectral efficient system. Despite all these benefits, OFDM faces some drawbacks: sensitivity to Doppler shift, and inefficient power consumption because of high PAPR [5]. Main aim of narrowband subcarrier is to obtain a channel which has almost constant band and also makes less complex equalization at the receiver.

\section{PERFORMANCE OF SC-FDMA}

SC-FDMA is mainly based on the single-carrier frequencydivision multiplexing (SC-FDM) modulation technique. It is also referred as discrete Fourier transform (DFT)-spread OFDM which is also a multiple access scheme. Its main performance is the same as for OFDM; thus, the same benefits are achievable in terms of multipath mitigation as well as lesscomplexity equalization [6]. But in SCFDMA, DFT is performed before the IFFT block, which spreads the data symbols over all the subcarriers containing information and produces virtual single-carrier structure. As a result, SC-FDM shows lower PAPR than OFDM [7].

This property makes SC-FDM attractive for uplink transmissions, as the user equipment (UE) benefits in terms of transmitted power efficiency. On one hand, all symbols are present in all subcarriers, DFT spreading allows the frequency selectivity of the channel to be exploited.

In OFDMA systems, sub carriers are allocated to multiple users. In uplink transmission, each terminal uses a subset of subcarriers to transmit its own data. Rest subcarriers that is not used for its own data transmission, will be filled with zeros. There are two ways of allocating subcarriers among all users: DFDMA (Distributed FDMA) and LFDMA (Localized FDMA). Here, DFDMA distributes M DFT outputs over the entire band (of total $\mathrm{N}$ subcarriers) with zeros filled in (N-M) unused subcarriers and Localized FDMA allocates DFT outputs to all $\mathrm{M}$ consecutive subcarriers in $\mathrm{N}$ number of subcarriers. When DFDMA distributes DFT output with equal distance $(\mathrm{N} / \mathrm{M}=\mathrm{S})$ it is known as IFDMA (Interleaved FDMA) where $\mathrm{S}$ is bandwidth spreading factor.

It will assume that the number of subcarriers allocated to each user is M. In the DFT-spreading technique, M-point DFT is used for spreading, and the output of DFT is assigned to the subcarriers of IFFT. The effect of PAPR reduction depends on the way of assigning the subcarriers to each terminal [8].
DFT Spreading for IFDMA, DFDMA and LFDMA: 3 users with $\mathrm{N}=12, \mathrm{M}=4$ and $\mathrm{S}=3$

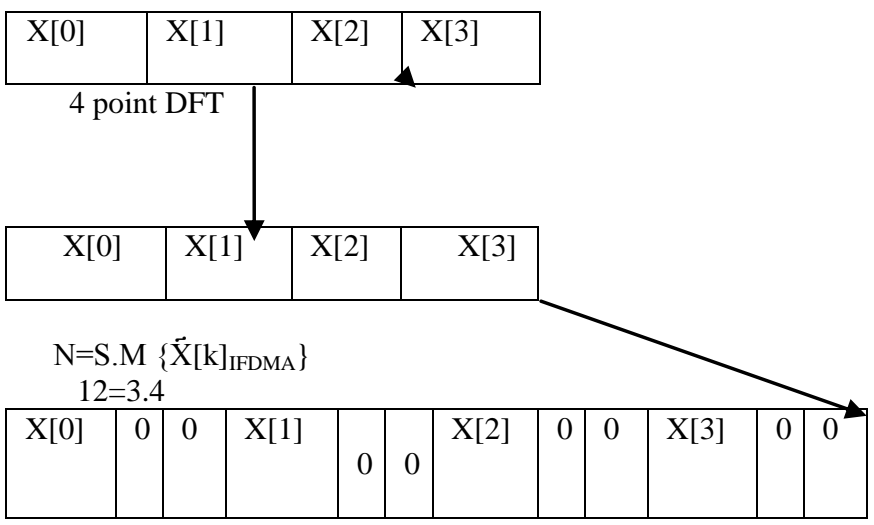

$\mathrm{N}>\mathrm{S} . \mathrm{M}\left\{\overrightarrow{\mathrm{X}}[\mathrm{k}]_{\text {DFDMA }}\right\}$

$12=3.4$

\begin{tabular}{|l|l|l|l|l|l|l|l|l|l|l|l|}
\hline $\mathrm{X}[0]$ & 0 & $\mathrm{X}[1]$ & 0 & $\mathrm{X}[2]$ & 0 & $\mathrm{X}[3]$ & 0 & 0 & 0 & 0 & 0 \\
\end{tabular}

$\mathrm{N}=\mathrm{S} . \mathrm{M}\{\overrightarrow{\mathrm{X}}[\mathrm{k}]$ LFDMA $\}$

$12=3.4$

\begin{tabular}{|l|l|l|l|l|l|l|l|l|l|l|l|}
\hline $\mathrm{X}[0]$ & $\mathrm{X}[1]$ & $\mathrm{X}[2]$ & $\mathrm{X}[3]$ & & & & & & & & \\
\end{tabular}

uplink transmitter with the DFT-spreading technique that employs IFDMA. Here, the input data $\mathrm{x}[\mathrm{m}]$ is DFT-spread to generate $\mathrm{X}[\mathrm{i}]$ and

then, allocated as

$\tilde{X}(k)=\left\{\begin{aligned} X\left[\frac{k}{s}\right], & k=S_{x} m 1_{x} m 1=0,1,2 . M-1 \\ 0_{x} & \text { otherwise }\end{aligned}\right.$

The IFFT $\tilde{x}[\mathrm{n}]$ with $\mathrm{n}=\mathrm{M} . \mathrm{s}+\mathrm{m}$ for $\mathrm{s}=0,1,2 \ldots ., \mathrm{S}-1$ and $\mathrm{m}==0,1,2 \ldots, \mathrm{M}-1$

$$
\begin{aligned}
\tilde{x}[\mathrm{n}]= & \frac{1}{N} \sum_{\mathbb{W}=0}^{N-1} \tilde{X}[k] \exp \left(\frac{\sqrt{2 \pi k m}}{N}\right) \\
& =\frac{1}{N} \frac{1}{S} \sum_{m 1=0}^{M-1} X[m 1] \exp ^{\mathrm{j} 2 \pi\left(\frac{n}{M} \mathrm{~m} 1\right)}
\end{aligned}
$$

Put the value of $n$ in (2.1)-

$=\frac{1}{5} \mathrm{x}[\mathrm{m}]$

Which turns out to be a repetition of the original input signal $\mathrm{x}[\mathrm{m}]$ scaled by $1 / \mathrm{S}$ in the time domain. In the IFDMA where the subcarrier mapping starts with the $r$ th subcarrier $(r=0,1$ ,2 ... S-1) ,the DFT spread symbol can be expressed as

$\tilde{X}(k)=\left\{\begin{aligned} X\left[\frac{k-r}{S}\right], & k=S . m 1+r_{*} m 1=0_{s} 1_{x} 2_{\alpha} M-1 \\ 0, & \text { otherwise }\end{aligned}\right.$

Then, the corresponding IFFT output sequence $\widetilde{x}[n]$, is given by 


$$
\begin{aligned}
& \tilde{x}[\mathrm{n}]=\tilde{\mathbb{X}}(M s+m) \\
& =\frac{1}{N} \sum_{\mathbb{k}=0}^{N-1} \hat{X}[k] \exp \left(\frac{j 2 \pi \mathrm{km}}{N}\right) \\
& =\frac{1}{S} \frac{1}{N} \sum_{k=0}^{N-1} X\left[m_{1}\right] \exp ^{\mathrm{j} 2 \pi\left(\frac{n}{M} \mathrm{~m} 1+\frac{n_{\mathrm{r}}}{N}\right)} \\
& =\frac{1}{5} \mathrm{x}[\mathrm{m}]_{\times} \exp ^{\mathrm{j} 2 \pi x} \frac{\mathrm{m}}{\mathrm{W}}
\end{aligned}
$$

Compared with (2.1), one can see that the frequency shift of subcarrier allocation starting point by $\mathrm{r}$ subcarriers results in the phase rotation of $\exp ^{j 2 \pi r} \frac{m i}{N}$ in IFDMA.

In the DFT-spreading scheme for LFDMA, the IFFT input signal $\tilde{X}(k)$ at the transmitter can be expressed as

$\tilde{X}(k)=\left\{\begin{array}{lc}X[k], & k=0,1,2 \ldots \ldots M-1 \\ 0 & k=M_{x} M+1 \ldots \ldots x-1\end{array}\right.$

Then, the corresponding IFFT output sequence

$$
\begin{aligned}
\tilde{X}[\mathrm{n}] & =\tilde{X}(M s+m)=\frac{1}{N} \sum_{k=0}^{N-1} \hat{X}[k] \exp \left(\frac{\sqrt{2 \pi k m}}{N}\right) \\
& =\frac{1}{S} \frac{1}{M} \sum_{k=0}^{N-1} X[k] \exp ^{j 2 \pi k \cdot \frac{S M+4}{S N}}
\end{aligned}
$$

For $\mathrm{s}=0 \mathrm{X}[\mathrm{k}]=\tilde{X}[5 m]$

$$
\begin{aligned}
& =\frac{1}{S} \frac{1}{M} \sum_{k=0}^{M-1} X[k] \exp ^{\mathrm{j} 2 \pi \mathrm{k} \frac{S \mathrm{~m}}{S M}} \\
& =\frac{1}{S} \frac{1}{M} \sum_{k=0}^{M-1} X[k] \exp ^{\mathrm{j} 2 \pi \mathrm{k}} \frac{\mathrm{m}}{M} \\
& =\frac{1}{5} \mathrm{x}[\mathrm{m}]
\end{aligned}
$$

For $\mathrm{s} \neq 0, \mathrm{X}[\mathrm{k}]=\sum_{p=0}^{M-1} x[p] \exp ^{-\mathrm{j} 2 \pi \mathrm{h} \frac{\mathbb{M}}{N}}$

$$
\begin{aligned}
& \widetilde{x}[\mathrm{n}]=\tilde{X}(S m+s)
\end{aligned}
$$

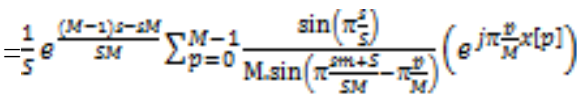

From (2.3) and (2.4) ,LFDMA signal becomes the 1/S-scaled copies of the input sequence at the multiples of $\mathrm{S}$ in the time domain. The values in-between are obtained by summing all the input sequences with the different complex-weight factor

\section{SIMULATION AND RESULTS}

Fig.2 shows a comparison of PAPR performances when the DFT-spreading technique is applied to the IFDMA, LFDMA, and OFDMA. Here, 4-QAM, 16-QAM, and 64-QAM are used for an SC-FDMA system with $\mathrm{N}=256, \mathrm{M}=64$, and $\mathrm{s}=4$. It can be seen from Fig. 2 that the PAPR performance of the DFTspreading technique varies depending on the subcarrier allocation method. In the case of 16-QAM, the values of PAPRs with IFDMA, LFDMA, and OFDMA for CCDF of $1 \%$ are $3.5 \mathrm{~dB}, 8.3 \mathrm{~dB}$, and $10.8 \mathrm{~dB}$, respectively. It implies that the PAPRs of IFDMA and LFDMA are lower by $7.3 \mathrm{~dB}$ and
$3.2 \mathrm{~dB}$, respectively, than that of OFDMA with no DFT spreading

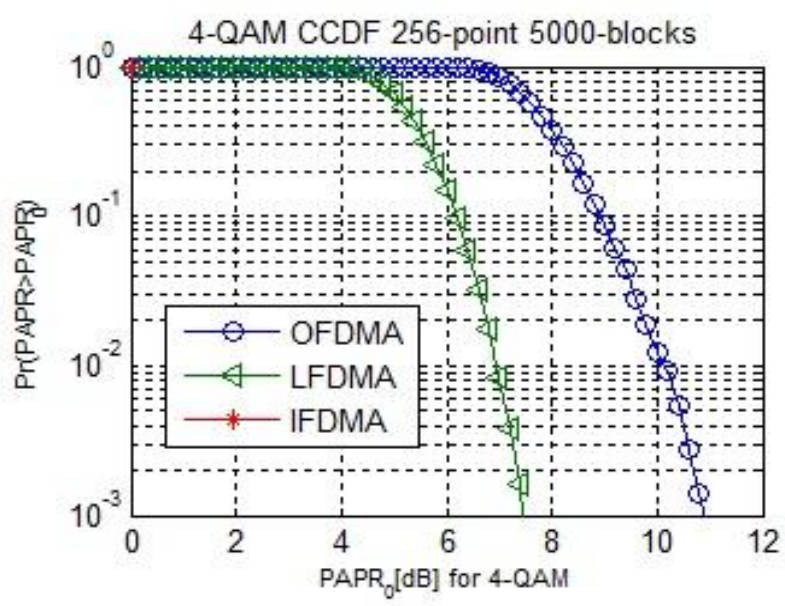

Fig.2-PAPR performances of DFT-spreading technique for IFDMA, LFDMA, and OFDMA with 4 QAM

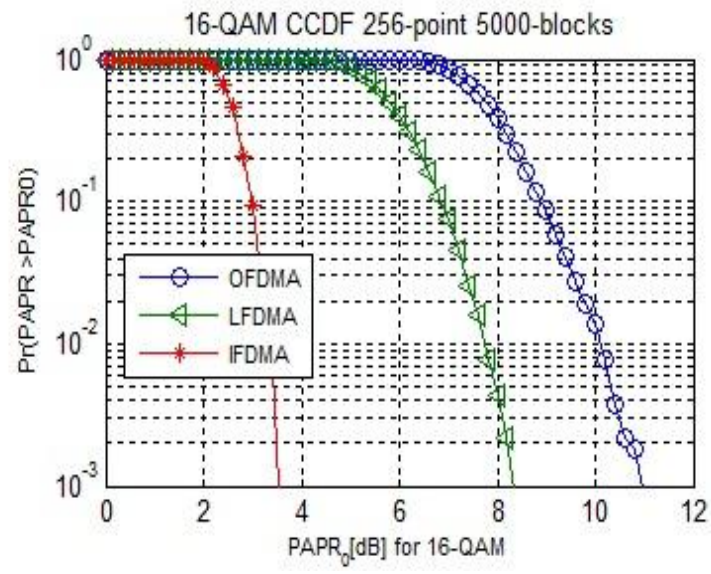

Fig.3-PAPR performances of DFT-spreading technique for IFDMA, LFDMA, and OFDMA with 16 QAM

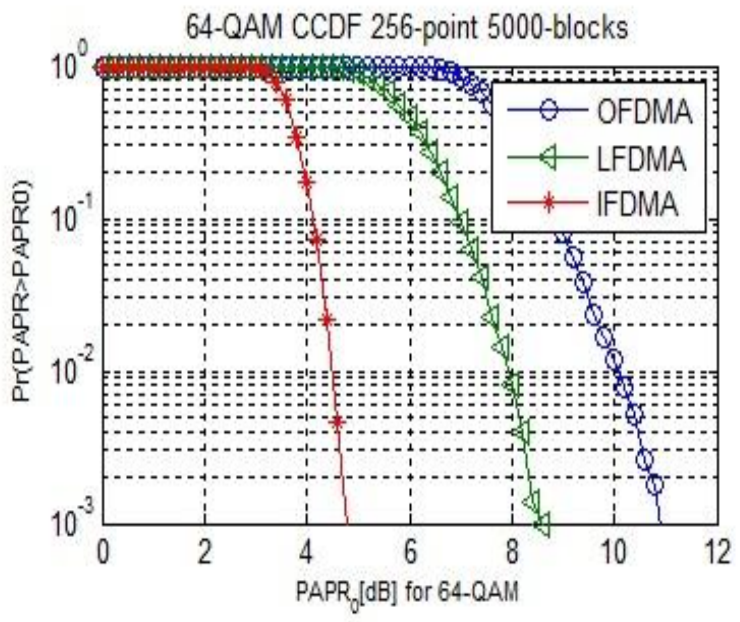

Fig.4-PAPR performances of DFT-spreading technique for IFDMA, LFDMA, and OFDMA with 64 QAM 


\subsection{Effect of pulse shaping}

In DFT-spreading scheme, PAPR performance is affected by pulse shaping. Figure 5 shows the PAPR graph of DFTspreading technique with IFDMA and LFDMA, varying independently with the roll-off factor $\alpha$ of the RC (RaisedCosine) filter for pulse shaping after the IFFT block. It has been observed from this figure that the PAPR graph of IFDMA can be significantly improved by increasing the rolloff factor from $\alpha=0$ to 1 whereas in LFDMA, PAPR is not so much affected by pulse shaping. It conclude that IFDMA will have a trade-off between excess bandwidth and PAPR graph since excess bandwidth increases as the roll-off factor becomes larger. Figure 3 shows the results with the simulation parameters of $\mathrm{N}=256, \mathrm{M}=64, \mathrm{~S}=4$ (spreading factor), and $\mathrm{No}=8$ (oversampling factor for pulse shaping) for both QPSK and 16-QAM.

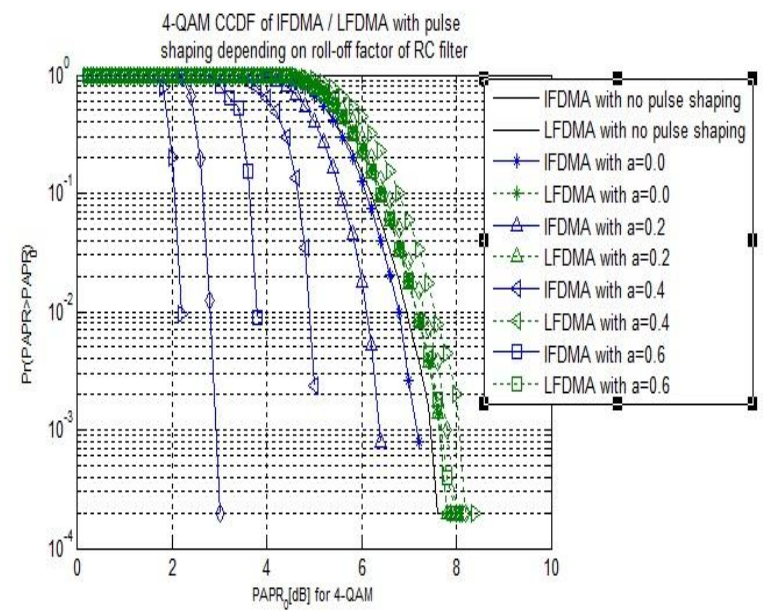

Fig.5-PAPR performances of DFT-spreading technique with pulse shaping in 4-QAM

\subsection{Effect of number of Subcarrier M:}

The PAPR graph of DFT- spreading technique is affected by changing the number of subcarriers, M. Fig. 4 shows that the PAPR graph of DFT-spreading technique for Localized FDMA with a roll-off factor of $\alpha=0.4$ is degraded as M increases, for example $\mathrm{M}=\mathrm{N}_{\mathrm{d}}=4$ to 128 . Here, 64-QAM is used for the SC-FDMA system with 256-point FFT ( $\mathrm{N}=256)$.

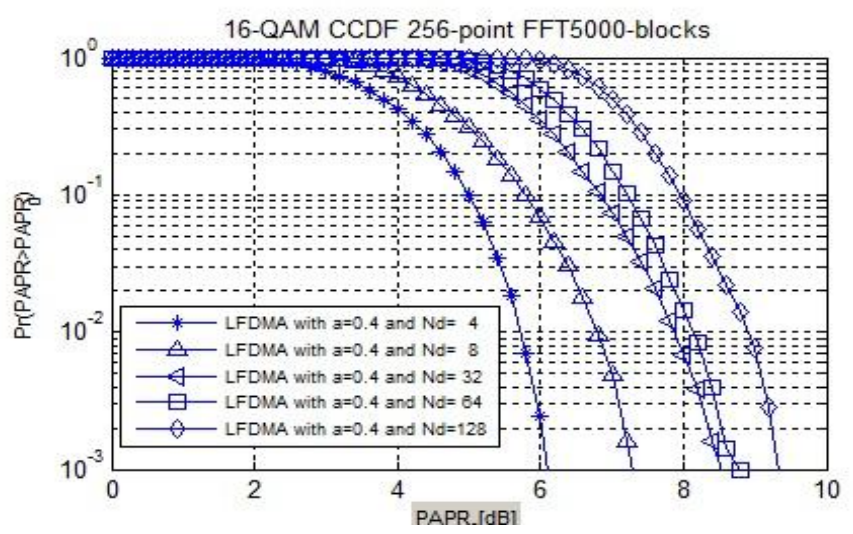

Fig.6-PAPR Reduction of DFT-spreading technique with variation of $\mathrm{N}_{d}$.

\section{CONCLUSION}

In conclusion, the PAPRs of LFDMA and IFDMA are lower by $3.2 \mathrm{~dB}$ and $7.3 \mathrm{~dB}$, respectively, than that of OFDMA of no DFT spreading. Results shows that, the SC-FDMA systems consisting of IFDMA and LFDMA have a better performance of PAPR than OFDMA systems.. Because of the fact that subcarriers allocation over the entire band (IFDMA) is not easy to implement and requires the additional resources such as guard band and pilots, hence LFDMA is preferred for implementation although IFDMA shows lower PAPR in above results

\section{REFERENCES}

[1] D. Falconer, S.L. Ariyavisitakul, A. Benyamin- Seeyar, and B. Eidson, "Frequency domain equalization for single-carrier broadband wireless systems,"IEEE Commun. Mag., vol. 40, no. 4, pp. 5866, Apr. 2002.

[2] H. Sari, G. Karam , and I. Jeanclaude, "Transmission Techniques for Digital Terrestrial TV broadcasting,"IEEE Commun. Mag., vol. 33, no. 2, pp. 100109, Feb. 1995.

[3] A. Czylwik, "Comparison between adaptive OFDM and single carrier modulation with frequency domain equalization," inProc. VTC'97 Phoenix, AZ, vol. 2, May 1997, pp. 865-869.

4] M. J. Hao and C. H. Lai, "Precoding for PAPR reduction of OFDM signals with minimum error probability," IEEE Transactions on Broadcasting, vol. 56, no. 1, pp. 120 128,2010

[5] L. Hanzo et al., Eds., "OFDM and MC-CDMA for Broadband Multi-User Communications, WLANs and Broadcasting," Wiley/IEEE Press, 2003.

[6] S. L. Falconer et al., "Frequency Domain Equalization for Single-Carrier Broadband Wireless Systems," IEEE Commun. Ma g., vol. 40, no. 4, Apr. 2002, pp. 58-66.

[7] Myung, Lim, and Goodmand, "Single Carrier FDMA for Uplink Wireless Transmission," IEEE Vehic. Tech. Mag., vol. 1, Sept. 2006, pp. 30-38.

[8] Myung, H.G., Lim, J., and Goodman, D.J. (2006) Single carrier FDMA for uplink wireless transmission .IEEE Veh. Technol. Mag., 1(3), 30-38.

[9] Yong Soo Cho, Jaekwon Kim, Won Young Yang, Chung G. Kang. Mimo -ofdm wireless communications with matlab.

[10] J. T. Mourelo, "PAPR Reduction for Multicarrier Modulation," PhD thesis, University of Stanford, 1999.

[11] 3GPP TSG RAN WG1 Mtg. 44 R1-060540, "Performance of Localized and Distributed SC-FDMA," Feb. 2006.

[12] W. Wang et al., "Impact of Multi-User Diversity and Channel Variability on Adaptive OFDM," IEEE Proc. $58^{\text {th }}$ VTC., vol. 1, Oct. 2003, pp. 547-51 